\title{
MAGNETIC ANNIHILATION \\ IN COLLIDING-WIND BINARY SYSTEMS
}

\author{
M. JARDINE 1 , H.R. ALLEN ${ }^{2}$ and A.M.T. POLLOCK ${ }^{3}$ \\ ${ }^{1}$ Astronomy Centre, University of Sussex, Brighton BN1 9QH, England \\ ${ }^{2}$ Computing Centre, North Haugh, St. Andrews, Fife KY16 9SX, Scotland \\ ${ }^{3}$ Computer \& Scientific Co. Ltd, 34 Westwood Road, Sheffield S11 7EY, England
}

\begin{abstract}
We investigate the possibility that a stagnation-point magnetic reconnection model may account for the particle acceleration necessary for the generation of nonthermal radio emission in the Wolf-Rayet binary systems exemplified by WR140.
\end{abstract}

Key words: reconnection - particle acceleration - radio: non-thermal - stars: Wolf-Rayet

\section{WR140's non-thermal radio emission}

One of the remarkable properties of the WR+O binary WR140 (e.g., Williams et al.1990) is the brightness and long-term variability of its nonthermal radio emission. The binary-orbit related modulation of the flux at a few $\mathrm{cm}$ clearly demonstrates that the radio source follows a path through the Wolf-Rayet wind similar in geometry to the orbit of the $\mathbf{O}$-star. It is natural to identify the seat of the radio emission to be in the region where the winds from the two stars collide, a conclusion supported by van der Hucht $e t$ al.'s (1992) VLBI measurements. However, despite the wealth of beautiful measurements, there are few ideas concerning the physics of the emission, although is likely to be synchrotron radiation, immediately implying the presence of relativistic electrons and a magnetic field. While the demonstration of the presence of a magnetic field in a hot-star wind is clearly of fundamental importance to the study of these objects, little or nothing is known of its origin and we take it as axiomatic. The other vital synchrotron ingredient is the relativistic electrons. Eichler \& Usov (1993) and Usov (these proceedings) have discussed the possibility of particle acceleration by the strong shocks present in colliding-wind binaries. We have investigated a different mechanism that is supposed to contribute to the acceleration of non-thermal particles in a variety of astrophysical settings such as, for example, at the earth's magnetopause and in solar flares, namely magnetic reconnection or annihilation.

\section{A magnetic annihilation particle acceleration model}

Colliding-wind binaries provide an almost text-book example of magnetic annihilation. We have taken a very simple-minded approach. The two stellar magnetic fields, that are likely to be azimuthal because of stellar rotation, 
are pushed together in the flow causing a current sheet to form near the stagnation point. Within the current sheet, an electric field is generated parallel to the magnetic field and is able to accelerate electrons to high speeds if collisional losses are overcome, a condition satisfied if the electric field exceeds the so-called Dreicer field, $E>E_{\mathrm{D}}$, where

$$
E_{\mathrm{D}}=\frac{12 \pi e^{3} n_{\mathrm{e}} \ln \Lambda}{m_{\mathrm{e}} v_{\mathrm{e}}^{2}}
$$

Here $v_{e}$ is the thermal electron speed, $n_{e}$, the electron density and $\ln \Lambda$ the Coulomb logarithm. This condition is easily met in WR140's collision region. For acceleration over a distance, $L$, the maximum energy is given by $\gamma_{\max } m_{\mathrm{e}} c^{2}=e E L$ where we have neglected all electron energy losses, including the synchrotron emission we are trying to estimate. In order to find $E$ and $B$ we have made numerical magnetohydrodynamic models of the flow only through the current sheet under the assumptions that the magnetic field lines are locally straight and vary across but not along the sheet; that the flow is axially symmetric and incompressible; and that there is no time dependence. The independent variables are $v_{\mathrm{w}}$, the speed of wind material flowing into the current sheet; $B_{\mathrm{w}}$, the local wind magnetic field; $L$, the length of the current sheet; and $R_{\mathrm{m}}$, the magnetic Reynolds number. The numerical solutions for $E$ and $B$ in the current sheet, and its width $l$, yield the following analytical approximations :

$$
l \sim 2 L R_{\mathrm{m}}^{-1 / 2} B \sim 2 B_{\mathrm{w}} R_{\mathrm{m}}^{1 / 4} E \sim 1.2 \times 10^{-4} v_{\mathrm{w}} B_{\mathrm{w}} R_{\mathrm{m}}^{-1 / 4}
$$

Inserting suitable values for WR140 $v_{\mathrm{w}}=v_{\infty}$ and $B_{\mathrm{w}}=B_{*}\left(r / R_{*}\right)^{-2} \mathrm{G}$, and taking $\varepsilon$ to be the fraction of electrons accelerated to $\gamma_{\max }$, yields the total synchrotron power

$$
P \sim \varepsilon\left(\frac{B_{*}}{1 \mathrm{G}}\right)^{5}\left(\frac{L}{10^{13} \mathrm{~cm}}\right)^{4}\left(\frac{R_{\mathrm{m}}}{10^{16}}\right)^{1 / 4} 10^{29} \mathrm{ergs} \mathrm{s}^{-1}
$$

Inserting van der Hucht et al.'s (1992) observed values for WR140 at $6 \mathrm{~cm}$ at maximum of $\nu f_{\nu} \approx 2.5 \times 10^{29} \mathrm{ergs} \mathrm{s}^{-1}$ and using $L \approx 1.3 \times 10^{14} \mathrm{~cm}$ from the VLBI measurements shows that for values of the surface field, $B_{*}$, of a few $\mathrm{G}$ and reasonable particle acceleration efficiency, $\varepsilon$, magnetic reconnection provides a viable explanation for the non-thermal radio emission from WR140 for high values of the magnetic Reynolds number $R_{\mathrm{m}} \approx 10^{16}$.

\section{References}

Eichler, D., Usov, V.V. 1993, ApJ 402, 271

van der Hucht, K.A., P.M. Williams, Spoelstra, T.A.Th., de Bruyn, A.G. 1992, in: L. Drissen, C. Leitherer \& A. Nota (eds.), Nonisotropic and Variable Outflows from Stars, ASP Conf. Series 22, 253

Williams, P.M., van der Hucht, K.A., Pollock, A.M.T., Florkowski, D.R., van der Woerd, H., Wamsteker, W.M. 1990, MNRAS 243, 662 